Mappemonde

Revue trimestrielle sur l'image géographique et les

formes du territoire

$124 \mid 2018$

Varia

J. DAVIES et A. J. KENT, The Red Atlas

Chicago et Londres : Chicago University Press, 234 p.

Laurent Jégou

(2) OpenEdition

Journals

Édition électronique

URL : http://journals.openedition.org/mappemonde/367

DOI : 10.4000/mappemonde.367

ISSN : 1769-7298

Éditeur

UMR ESPACE

Référence électronique

Laurent Jégou, « J. DAVIES et A. J. KENT, The Red Atlas », Mappemonde [En ligne], 124 | 2018, mis en ligne le 10 juillet 2018, consulté le 25 septembre 2020. URL : http://journals.openedition.org/mappemonde/ 367 ; DOI : https://doi.org/10.4000/mappemonde.367

Ce document a été généré automatiquement le 25 septembre 2020.

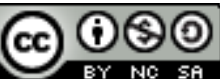

La revue Mappemonde est mise à disposition selon les termes de la Licence Creative Commons Attribution - Pas d'Utilisation Commerciale - Partage dans les Mêmes Conditions 4.0 International. 


\section{J. DAVIES et A. J. KENT, The Red Atlas}

Chicago et Londres : Chicago University Press, 234 p. Laurent Jégou

\section{RÉFÉRENCE}

J. DAVIES et A. J. KENT (2017). The Red Atlas. Chicago et Londres : Chicago University Press, $234 \mathrm{p}$.

Site Internet compagnon : https://redatlasbook.com 
1 The Red Atlas est l'histoire d'un programme de cartographie très particulier, qui a étonné et intéressé de nombreux géographes, cartographes ou simples amateurs de cartes lors de sa découverte et sa révélation au public: les «cartes secrètes» produites par l'URSS sur une grande partie du territoire mondial et notamment les pays et villes « occidentaux». Les auteurs sont John Davies, collectionneur et spécialiste de cette source, et Alexander J. Kent, enseignant-chercheur à la Canterbury Christ Church University qui en a fait un de ses objets de recherche.

2 Parce que cet ouvrage s'intéresse principalement à la production de ces

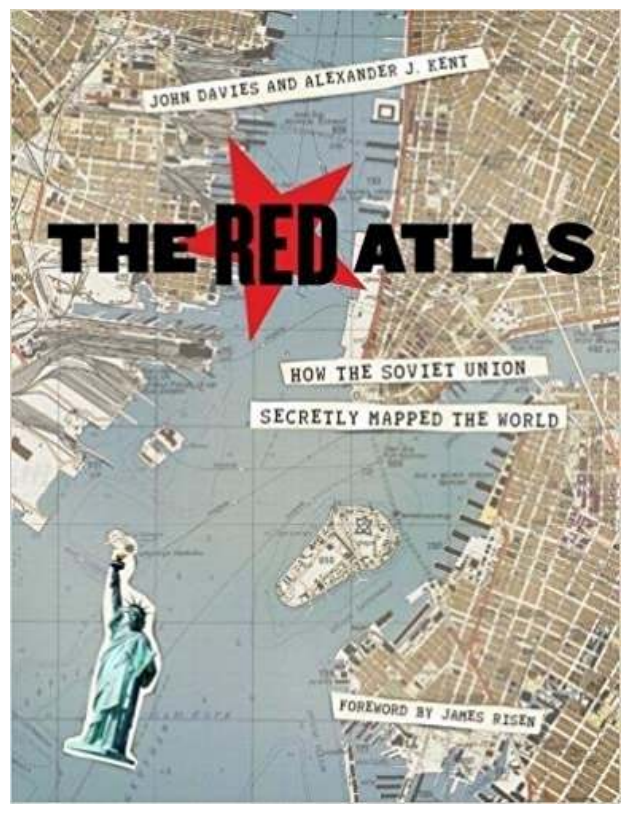
cartes et à ce qu'elles disent de la guerre froide, les auteurs ne décrivent les conditions de leur mise au jour qu'en dernière partie (p. 131, « Resurrection »), mais il est utile d'y revenir rapidement.

3 À partir de la chute du Mur de Berlin en 1989 puis de celle l'URSS en 1991, lors de la désaffection progressive des anciennes bases militaires soviétiques, le matériel qui s'y trouvait s'est progressivement retrouvé sur le marché, vendu par les officiers responsables eux-mêmes. 25 de ces bases comprenaient le dépôt d'un jeu complet de cartes topographiques du monde et de grandes villes. Comme on le verra, ces cartes possédaient une valeur importante, elles ont fait partie des échanges qui ont progressivement vidé les bases militaires contre des devises fortes, par des canaux d'une légalité très variable, mais souvent douteuse. Des libraires et collectionneurs, essentiellement anglais et nord-américains, puis des cartothèques universitaires, se sont intéressés à ces cartes, mais aussi des ONG devant intervenir sur le terrain dans des régions peu cartographiées, ainsi que les militaires eux-mêmes, notamment lorsque les cartes des territoires de l'ex-URSS et de l'Afghanistan furent disponibles. La « libération » de près de 6000 tonnes de cartes du dépôt de Riga, vendues au poids du papier par les officiers de la base, fut notamment l'occasion de diffuser des documents cartographiques inédits sur les régions russes (au grand étonnement des ex-officiers du KGB qui les découvrirent dans une foire aux livres en Allemagne...).

4 L'ouvrage débute par la présentation de l'histoire et du contexte de la production cartographique militaire en URSS, pour se focaliser sur le programme des «cartes secrètes ", tel qu'on l'a progressivement découvert. L'URSS a mis en œuvre un projet de cartographie topographique détaillée de son territoire dès 1918, d'abord à petite échelle (millionième), dans le but de mettre à jour la première carte topographique impériale qui datait de 1801. Ce programme s'est progressivement étendu, notamment pendant la $2^{\mathrm{e}}$ guerre mondiale, sur la Pologne et le front oriental. Après la guerre, les opérations cartographiques secrètes s'intéressent aux principales villes occidentales, à des échelles assez grandes (1/10 000 et 1/25 000), selon des spécifications rigoureuses pour garantir une précision et une légende homogène et en couleurs. 
5 Alors que les cartes officielles soviétiques destinées au public restent à une très petite échelle $(1 / 2,5 \mathrm{M})$ et sont volontairement parsemées d'erreurs, la Direction de la Cartographie Militaire de l'État-Major de l'Armée Soviétique s'engage dans un programme dont le gigantisme reste à cerner précisément, mais dont on estime que la production va dépasser le million de cartes.

The Red Atlas décrit ensuite dans le détail le contenu des cartes elles-mêmes et les hypothèses développées à propos de leur conception. Au-delà de son aspect quantitatif, l'effort de cartographie "secrète " russe impressionne par sa sophistication: une légende incroyablement détaillée, basée sur un vocabulaire de plusieurs centaines de symboles (les auteurs parlent du système de légende topographique le plus étendu jamais mis au point cf. figure 1), ce qui implique une précision et une profondeur des sources d'information utilisées qui laissent songeur quant à la motivation et aux moyens de renseignement déployés. Le revers des cartes à grande échelle propose un texte descriptif, la «spravka » (СПРАВКА légende détaillée), à propos des types d'activités et des niveaux d'intérêt stratégiques des lieux repérés par des points numérotés.

Figure 1. Extrait de la légende des cartes à grande échelle, hydrographie, les ponts.

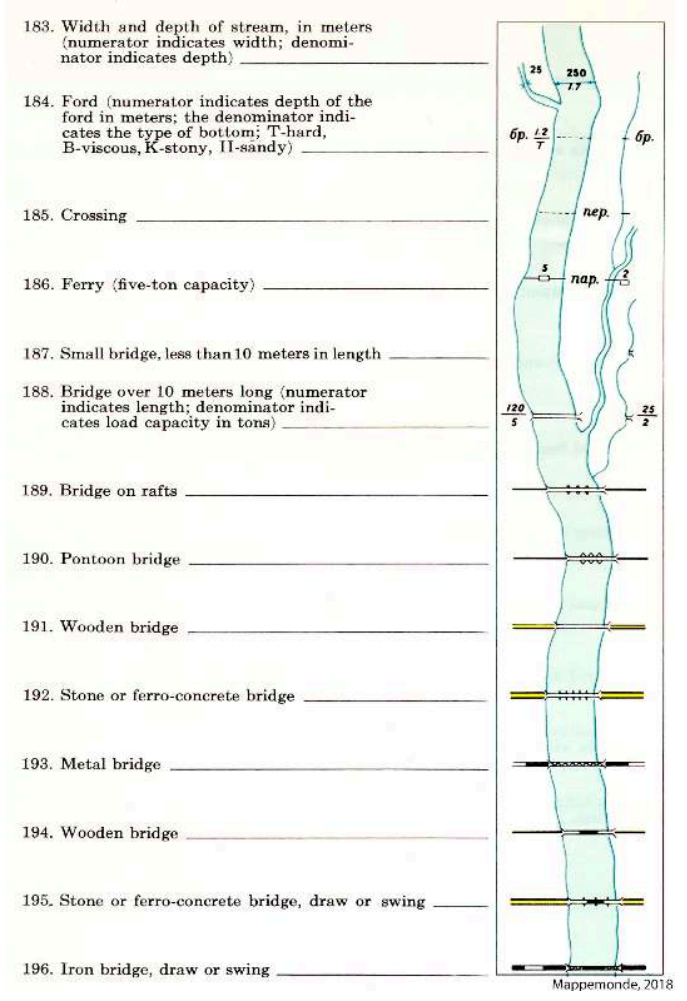

7 Naturellement, un effort secret de cartographie militaire, précis et étendu à des régions très éloignées du territoire national, ne peut qu'évoquer une velléité d'invasion ou de bombardement. On le remarque particulièrement au travers de la précision de toutes les informations concernant la circulation, le déplacement de troupes et de matériel (figure 2). 
Figure 2. Extrait de la carte au 1/50 000 d'Oxford, Angleterre.

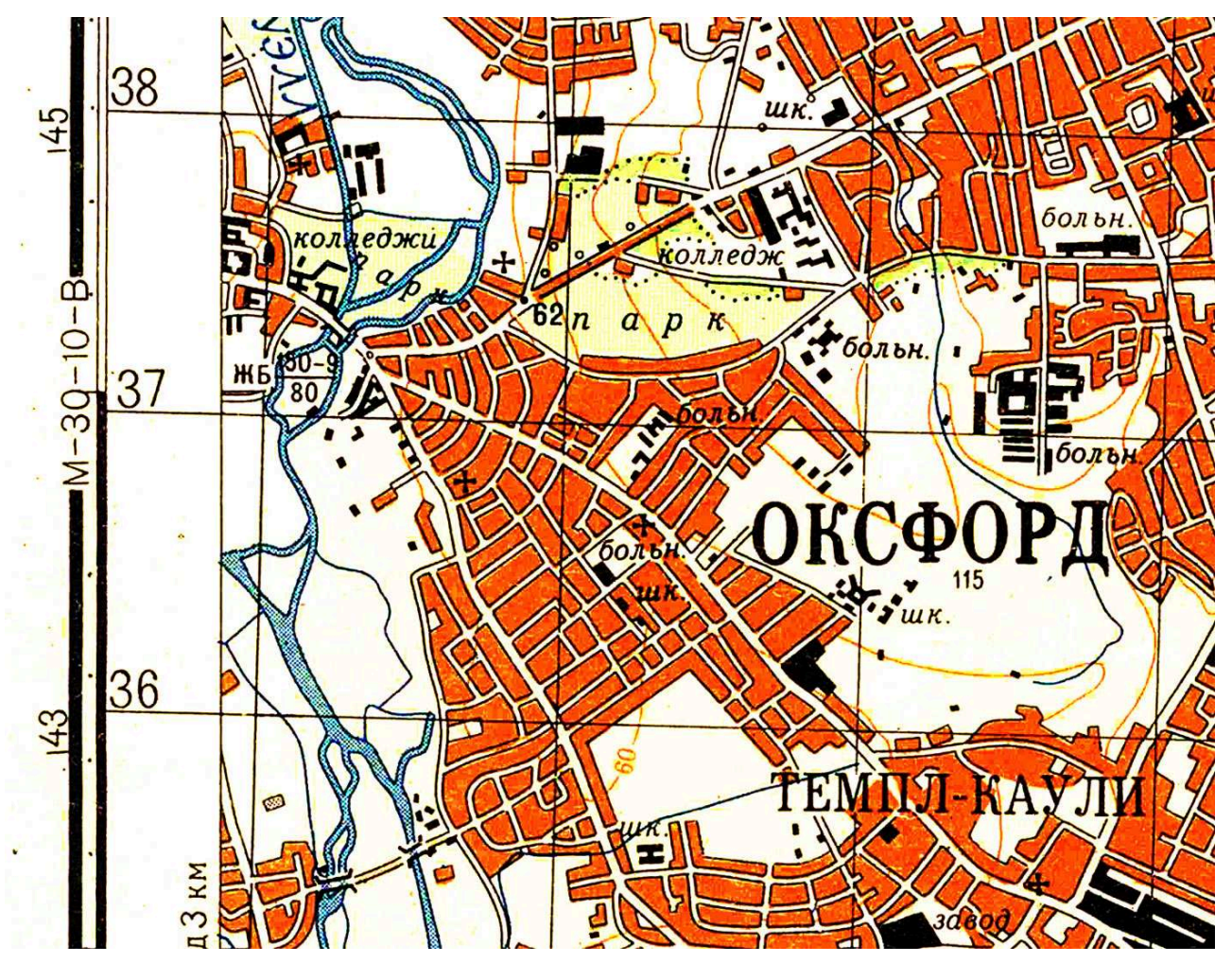

De même, toutes les activités «stratégiques » sont nettement identifiées, notamment les entreprises participant à l'effort nucléaire étranger, les ressources minières, les ports et les aéroports, ainsi que tous les terrains militaires connus. Les auteurs relèvent souvent la surprise de certains habitants en repérant sur ces cartes des adresses d'entreprises dont les activités n'étaient pas de nature publique, comme des fabricants de torpilles sous-marines à Seattle (figure 3)... 
Figure 3. Extrait de la carte « secrète » de Seattle au 1/25 000.

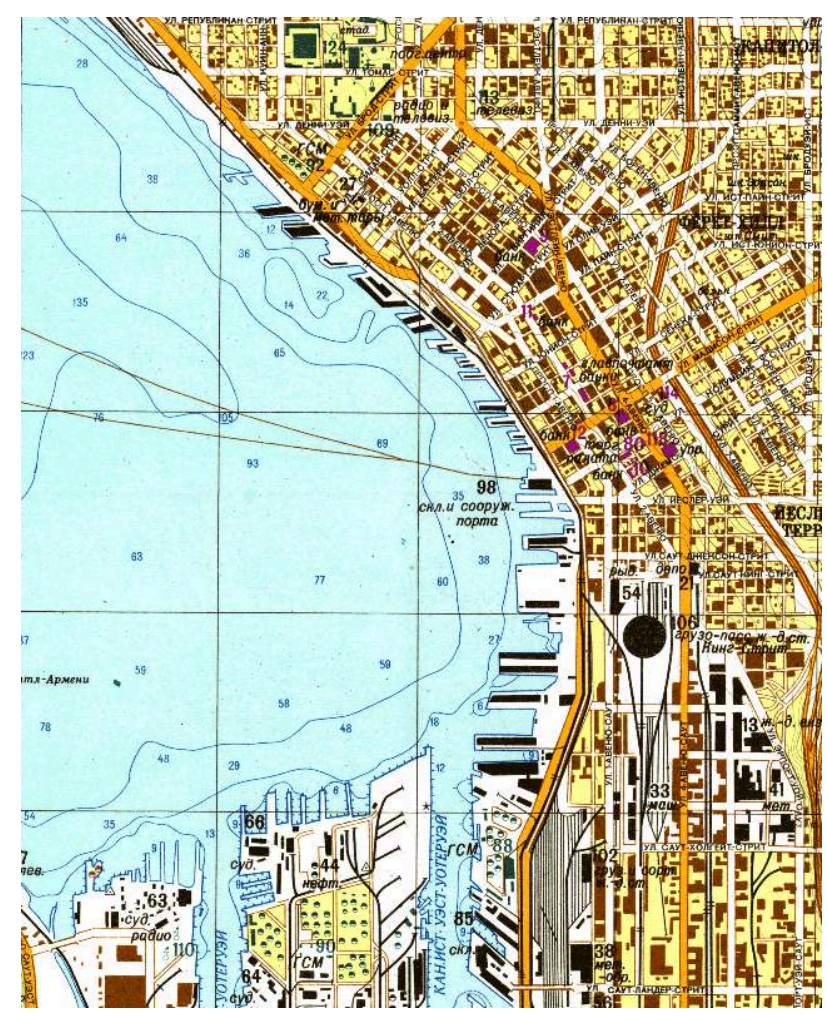

9 Les sources d'information utilisées pour la production de ces cartes secrètes furent nombreuses, diversifiées, l'ouvrage expose les différentes hypothèses émises quant à ces sources en comparant les cartes avec celles de référence pour la zone et l'époque. Ainsi, une grande part des informations provient des cartes topographiques officielles disponibles, généralement publiques. Cependant, les différences sont remarquables, les cartes secrètes étant souvent bien plus précises et mieux renseignées (on note toutefois quelques erreurs d'interprétation et exagérations). Les deux principales sources d'information qui alimentèrent ces cartes furent les programmes d'observation par satellite et avions-espions (notamment la série des satellites Zenith à partir de 1962), ainsi que le travail de terrain clandestin, nous sommes ici en plein dans l'imaginaire de l'espionnage pendant la guerre froide. Des informations comme la disposition de certains camps militaires ou de sites industriels stratégiques, la praticabilité des forêts par des engins à chenilles (écart entre les arbres, type de sol), les profondeurs des canaux ou l'état réel des chemins, indiscernables sur les images aériennes, ne pouvaient être acquises que par des agents sur place (figure 4). 
Figure 4. Extrait de la carte au 1/200 000 de Boulogne, Calais, 1964.

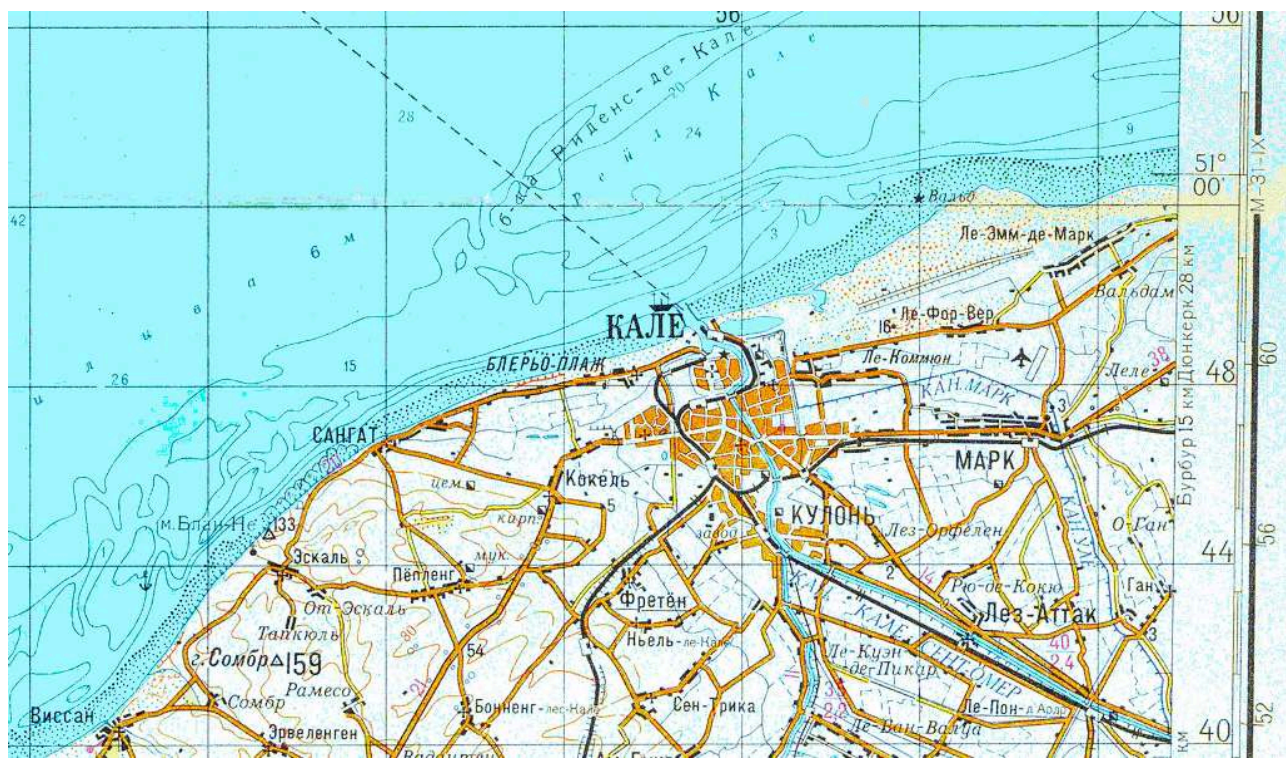

L'ouvrage se termine par une importante série d'annexes, dont une cinquantaine de reproductions de cartes, des extraits de légendes et un guide de traduction des acronymes récurrents.

11 The Red Atlas permet donc de découvrir et de comprendre le programme de cartographie «secrète » engagée pendant la guerre froide par l'URSS, à la fois sur le plan de la forme, des techniques cartographiques (généralisation, légende), mais aussi dans ses aspects géopolitiques et de renseignement. Très largement illustré, l'ouvrage présente à ses lecteurs de nombreuses reproductions, extraits, détails des cartes, qui viennent utilement compléter les descriptions. La surprise et l'effet d'étrangeté de ces cartes de pays "occidentaux» en cyrillique sont dépassés et approfondis par une discussion scientifique, cartographique, géographique et géopolitique... sérieusement argumentée.

\section{AUTEURS}

\section{LAURENT JÉGOU}

Université de Toulouse-Jean Jaurès / UMR LISST-Cieu 